\title{
Power-Splitting Scheme for Nonlinear Energy Harvesting AF Relaying with Direct Link
}

\author{
Xiaobo Bai $(\mathbb{D}),{ }^{1}$ Jingfeng Shao, ${ }^{1}$ Jiangang Tian, $^{2}$ and Liqin Shi ${ }^{1}{ }^{1}$ \\ ${ }^{1}$ School of Management, Xian Polytechnic University, Xi'an, China \\ ${ }^{2}$ Army Academy of Border and Coastal Defence, Department of Information and Arms, Xian, China \\ Correspondence should be addressed to Liqin Shi; connectliqin@126.com
}

Received 3 May 2018; Revised 6 June 2018; Accepted 10 June 2018; Published 2 July 2018

Academic Editor: Fuhui Zhou

Copyright (c) 2018 Xiaobo Bai et al. This is an open access article distributed under the Creative Commons Attribution License, which permits unrestricted use, distribution, and reproduction in any medium, provided the original work is properly cited.

\begin{abstract}
Simultaneous wireless information and power transfer (SWIPT) is a promising technique to prolong the lifetime of energyconstrained relay systems. Most previous works optimize power-splitting (PS) scheme based on a linear or a simple two-piecewise linear energy harvesting (EH) model, while the employed EH model may not characterize the properties of practical EH harvesters well. This leads to a mismatch between the existing PS scheme and the practical EH harvester available for relay systems. Motivated by this, this paper is devoted to the design of PS scheme in a nonlinear EH amplify-and-forward energy-constrained relay system in the presence of a direct link between the source and the destination. In particular, we formulate an optimization problem to maximize the system capacity according to the instantaneous channel state information, subject to a nonlinear EH model based on the logistic function. The objective function of the formulated problem is proven to be unimodal and there is no closed-form expression for the optimal PS ratio due to the complexity of logistic function. In order to reduce overhead cost of optimizing PS ratio, a simpler nonlinear EH model based on the inverse proportional function is employed to replace the nonlinear EH model based on the logistic function and we further derive the closed-form expression for the optimal PS ratio. Simulation results reveal that a higher system capacity can be achieved when the PS scheme is optimized based on nonlinear EH models instead of the linear EH model, and that there is only a marginal difference between the capacity under the two optimal PS schemes optimized for two different nonlinear EH models.
\end{abstract}

\section{Introduction}

The aim of Internet of Things (IoT) is to enable people and things to be connected anytime, anyplace, with anything and anyone, ideally using any path/network and any service [1]. It allows massive IoT devices such as low-power wireless sensors to access the wireless communication networks and communicate with each other. The limited lifetime of IoT devices is a fundamental problem for implementing IoT deployment. Motivated by this, simultaneous wireless information and power transfer (SWIPT) is proposed and devoted to the solution of this problem. On the other hand, relaying technology is highly beneficial in wireless communications in terms of the communication range, the energy efficiency, and the system capacity $[2,3]$. However, when the relaying technology is employed in IoT networks, the IoT devices are unwilling to be a relay in order to avoid the extra energy consumption since these devices usually have limited battery capacity. Thus, integrating SWIPT and relaying is a viable option to strike a tradeoff between information processing and power supply and gains much attention recently [4-6]. In this field, the design of energy harvesting (EH) scheme, which instructs the relay either to switch the received radio frequency $(\mathrm{RF})$ signal in the time domain or to split the received RF signal in the power domain to provide signal processing and power transfer, is one of the most important issues.

Until now, there have been many reports [4-14] regarding how to design an appropriate EH scheme for SWIPT based relay systems. The works $[4,7]$ studied the effects of power-splitting (PS) ratio and time-switching (TS) ratio on the amplify-and-forward (AF) and decode-and-forward (DF) relay systems by deriving the expressions for outage probability and ergodic capacity. In [8], both the PS ratio and 
TS ratio were optimized to maximize the system transmission rate in DF relay systems, where the relay has a certain amount of remaining energy. By combining both TS and PS, a hybrid scheme was proposed and further optimized in [9]. The authors of [10] designed two optimal PS schemes with full and partial channel state information (CSI) to minimize the system outage probability in an AF relay system. Assuming the availability of source-destination link, the optimal PS ratio was designed and the diversity gains for the relay and the destination were analyzed [11]. By means of the stochastic geometry, the authors proposed a dynamic PS scheme in a DF relay system with a random number of transmitter-receiver pairs and investigated its outage probability [12]. Recognizing the advantages of nonorthogonal multiple access (NOMA) in spectrum efficiency, a novel cooperative SWIPT-NOMA system was integrated [13], and an optimal PS scheme was further proposed [14].

These works [4-14] were based on a linear EH model, in which the RF-to-direct current (DC) power conversion efficiency is a fixed constant and independent of the input power of the energy harvester. As pointed out by [15-17], the practical energy harvester operates in a nonlinear mode and the linear $\mathrm{EH}$ model may not characterize the properties of practical EH harvesters well. Further, the optimal PS schemes based on the linear EH model may not be optimal for the practical scenario. As a result, the existing schemes based on a linear EH model may need to be redesigned to avoid the mismatch caused by the resource allocation under the linear $\mathrm{EH}$ model, and ever-increasing attention has been paid into the study of nonlinear $\mathrm{EH}$ model in wireless communications (see [18-30] and references therein). References [18-25] introduced the nonlinear $\mathrm{EH}$ model into the wireless powered communication networks, the SWIPT-NOMA system, and the cognitive radio networks with SWIPT, where the resource allocation scheme, including the transmit power of the transmitter, and the PS/TS ratio, is concentrated. The studies revealed that a higher system capacity could be achieved by designing the $\mathrm{EH}$ scheme based on the nonlinear $\mathrm{EH}$ model instead of the conventional linear one. Apart from the aforementioned networks, the researchers have also studied the design of $\mathrm{EH}$ scheme in nonlinear $\mathrm{EH}$ relay systems [26-30]. For example, the authors of [26, 27] focused on the design of PS scheme for nonlinear EH two-way relay systems. Since the low complexity of hardware is very vital to energy-constrained relay systems, the researches on oneway relay systems have attracted a lot of interests [28-30]. In particular, the works $[28,29]$ derived the outage probability of a PS enabled nonlinear EH relay system. Considering the perfect/imperfect CSI at the relay, an optimal PS scheme was developed to minimize the outage probability [30] in an AF nonlinear EH relay system. These aforementioned works have laid the foundation for the design of $\mathrm{EH}$ scheme in one-way relay systems. After careful analysis of the existing works [28-30], it can be found that a simple two-piecewise linear EH model was employed, and that the employed twopiecewise linear model cannot provide sufficient precision compared with the existing nonlinear EH models based on the logistic function and the inverse proportional function. Therefore, there still remains a large gap to be filled regarding the design of $\mathrm{EH}$ scheme for nonlinear $\mathrm{EH}$ one-way relay systems.

Motivated by this observation, this paper is devoted to the design of PS schemes for an AF relay system with direct link in terms of system capacity, where the nonlinear $\mathrm{EH}$ models proposed in $[16,18]$ are used to characterize the properties of practical EH circuits. Our contributions are as follows.

(i) We optimize the PS scheme to maximize the system capacity under the nonlinear $\mathrm{EH}$ model based on a logistic function. We prove that the objective function is unimodal and the optimal solution is obtained by the golden section search method.

(ii) Employing the nonlinear $\mathrm{EH}$ model based on an inverse proportional function instead of the logistic function, a closed-form expression for the optimal PS ratio is derived to maximize the system capacity. Compared with the PS scheme optimized for the nonlinear EH model based on the logistic function, the PS scheme optimized for the nonlinear EH model based on the inverse proportional enjoys a lower computational complexity with the near-optimal performance.

It is worth pointing out that energy efficiency (EE) is also an important performance metric. Since the EE is defined as the ratio of system capacity to power consumption [31], the optimization of EE is equivalent to the optimization of system capacity for a fixed transmit power. Thus, our derived optimal solution of this paper is the same as the optimal solution to maximize EE. If the transmit power is adjustable and smaller than a maximum power transmit, we should optimize both PS ratio and transmit power simultaneously. In this case, the solution to maximize EE is different from the derived results of this paper, while how to obtain the optimal PS ratio and optimal transmit power is beyond the scope of this paper.

\section{System Model and Working Flow}

As shown in Figure 1, we consider a SWIPT based AF relay system, composed of a source node $S$, an energy-constrained relay node $R$, and a destination node $D$. To be general, we assume that there exists a direct link between the source and the destination. All nodes operate in a half-duplex mode and are equipped with single antenna. It is assumed that both the source and the destination are equipped with fixed power supply, and that both "harvest-then-forward" scheme and the PS scheme are employed to encourage the relay to be cooperative with the source's transmission. Let $h_{i j}, \forall i, j \in$ $\{S, R, D\}$ and $i \neq j$ denote the channel coefficients between $i$ and $j$ in a quasistatic fading model. Let $P_{s}$ denote the transmit power of the source. All the channel state information (CSI) is available at the relay in order to investigate the system performance limits of the PS scheme. Moreover, we ignore the processing energy required by the transmit/receive circuitry at the relay [4-14]. 




Figure 1: An illustration of PS scheme.

2.1. Energy Harvesting Model. Since the conventional linear EH model may not be accurate for modelling the practical energy harvester due to the nonlinearity of the diodes, inductors, and capacitors, by fitting over real measurement data based on the logistic function, the authors of [18] proposed an accurate nonlinear $\mathrm{EH}$ model, given by

$$
P_{\mathrm{H}}^{(1)}=\frac{E_{\mathrm{max}} /\left(1+\exp \left(-a\left(P_{\mathrm{RF}}-b\right)\right)\right)-E_{\max } \Omega}{1-\Omega}
$$

where $P_{\mathrm{H}}^{(1)}$ denotes the amount of harvested energy; $P_{\mathrm{RF}}$ is the input power of the energy harvester; $a$ and $b$ are the parameters determined by the resistance, capacitance, and so on; $E_{\max }$ is the maximum harvestable power when the circuit becomes saturated and $\Omega=1 /(1+\exp (a b))$.

Recently, using the inverse proportional function instead of the logistic function to fit over real measurement data, Chen et al. in [16] proposed a more mathematically tractable nonlinear $\mathrm{EH}$ model, given as

$$
P_{\mathrm{H}}^{(2)}=\frac{a P_{\mathrm{RF}}+b}{P_{\mathrm{RF}}+c}-\frac{b}{c}
$$

where $a, b$, and $c$ are the fixed parameters. The comparison between the above two nonlinear models will be presented in Section 4 .

2.2. Working Flow. In the first time slot, $S$ broadcasts information $x_{s}$ to $R$ and $D$ with a transmit power $P_{s}$. Then the received signals at the relay and the destination node $D$ are given by

$$
\begin{aligned}
& y_{s r}=\sqrt{P_{s}} h_{S R} x_{s}+n_{s r} \\
& y_{s d}=\sqrt{P_{s}} h_{S D} x_{s}+n_{s d}
\end{aligned}
$$

where $\mathbb{E}\left\{\left|x_{s}\right|^{2}\right\}=1$ and $n_{s r} \sim \mathscr{C} \mathcal{N}\left(0, \sigma_{s r}^{2}\right)$ is the additive white Gaussian noise (AWGN) and $n_{s d} \sim \mathscr{C} \mathscr{N}\left(0, \sigma_{s d}^{2}\right)$.

For the destination $D$, the received signal-to-noise ratio (SNR) can be calculated as

$$
\gamma_{s d}=\frac{P_{s}\left|h_{S D}\right|^{2}}{\sigma_{s d}^{2}}
$$

For the relay $R$, after receiving the signal from the source $S$, the relay $R$ splits it into two parts: $\sqrt{\lambda} y_{s r}$ for harvesting energy and $\sqrt{1-\lambda} y_{s r}$ for information processing, where $\lambda$ is the PS ratio at the relay. Thus, the received RF power used for energy harvesting, $P_{\mathrm{RF}}$, and the signal used for amplification, $x_{r}$, are given by

$$
\begin{aligned}
P_{\mathrm{RF}} & =\lambda\left(P_{s}\left|h_{\mathrm{SR}}\right|^{2}+\sigma_{s r}^{2}\right) \\
x_{r} & =\sqrt{1-\lambda}\left(\sqrt{P_{s}} h_{S R} x_{s}+n_{s r}\right)+n_{p}
\end{aligned}
$$

where $n_{p} \sim \mathscr{C} \mathscr{N}\left(0, \sigma_{p}^{2}\right)$ is the additive white baseband Gaussian noise.

If the energy harvesting is completed, the total harvested energy is given by

$$
E_{\text {total }}=\frac{T}{2} P_{\mathrm{H}}
$$

where $P_{\mathrm{H}}$ is the output power of the energy harvester and $P_{\mathrm{H}}= \begin{cases}P_{\mathrm{H}}^{(1)}, & \text { if the non-linear EH model based on the logistic function is employed } \\ P_{\mathrm{H}}^{(2)}, & \text { if the non-linear EH model based on the inverse proportional function is employed. }\end{cases}$ 
In the second time slot, $R$ amplifies the signal $x_{r}$ by using the harvested energy and forwards the amplified signal to $D$. Hence, the received signal at $D$ is given by

$$
y_{r d}=h_{R D} G x_{r}+n_{r d}
$$

where $G=\sqrt{P_{\mathrm{H}} /\left((1-\lambda)\left(P_{s}\left|h_{S R}\right|^{2}+\sigma_{s r}^{2}\right)+\sigma_{p}^{2}\right)}$ is the amplifier gain at $R$ and $n_{r d} \sim \mathscr{C} \mathcal{N}\left(0, \sigma_{r d}^{2}\right)$ is the AWGN at $D$.

Then the received SNR from $R$ to $D$ is given by

$$
\begin{aligned}
& \gamma_{r d} \\
& =\frac{(1-\lambda) P_{s} P_{\mathrm{H}}\left|h_{S R}\right|^{2}\left|h_{R D}\right|^{2}}{P_{\mathrm{H}}\left|h_{R D}\right|^{2}\left((1-\lambda) \sigma_{s r}^{2}+\sigma_{p}^{2}\right)+\sigma_{r d}^{2}\left((1-\lambda) K+\sigma_{p}^{2}\right)}
\end{aligned}
$$

where $K=P_{s}\left|h_{S R}\right|^{2}+\sigma_{s r}^{2}$.

By implementing the maximal ratio combining (MRC) scheme at the destination $D$, the overall system capacity is given by

$$
C_{\text {total }}=\frac{T}{2} \log _{2}\left(1+\gamma_{s d}+\gamma_{r d}\right) .
$$

\section{Optimal Power-Splitting Scheme}

3.1. Nonlinear EH Model Based on the Logistic Function. In this subsection, we design an optimal PS scheme to maximize the overall system capacity for an AF relay system with SWIPT based on the nonlinear EH model in [18].

Substituting (1) into (11), the received SNR from $R$ to $D$ under the nonlinear EH model, in (1), is

$$
\gamma_{r d}^{(1)}=\frac{A(1-\lambda) P_{\mathrm{H}}^{(1)}}{B\left[(1-\lambda) P_{\mathrm{H}}^{(1)}+C P_{\mathrm{H}}^{(1)}+D_{1}(1-\lambda)+E\right]}
$$

where $A=P_{s}\left|h_{S R}\right|^{2}, B=\sigma_{s r}^{2}, C=\sigma_{p}^{2} / \sigma_{s r}^{2}, D_{1}=$ $K \sigma_{r d}^{2} /\left|h_{R D}\right|^{2} \sigma_{s r}^{2}$, and $E=\sigma_{r d}^{2} \sigma_{p}^{2} /\left|h_{R D}\right|^{2} \sigma_{s r}^{2}$.

Then the system capacity in this case can be computed as

$$
C_{\text {total }}^{(1)}=\frac{T}{2} \log _{2}\left(1+\gamma_{s d}+\gamma_{r d}^{(1)}\right) .
$$

Based on (14), the optimization problem can be formulated as

$$
\begin{aligned}
\mathscr{P} 1: \text { maximize } & C_{\text {total }}^{(1)} \\
\text { subject to } & 0 \leq \lambda \leq 1 .
\end{aligned}
$$

It is obvious that the optimization problem of maximizing the overall system capacity is equivalent to maximizing the received SNR from $R$ to $D$, which can be reformulated as

$$
\begin{aligned}
\mathscr{P} 2: \text { maximize } & \gamma_{r d}^{(1)} \\
\text { subject to } & 0 \leq \lambda \leq 1 .
\end{aligned}
$$

By taking the first-order derivative of the objective function $\gamma_{r d}^{(1)}$ with respect to $\lambda$, we have

$$
\frac{\partial \gamma_{r d}^{(1)}}{\partial \lambda}=\frac{-A f_{1}(\lambda) f_{2}(\lambda)}{B\left[(1-\lambda) P_{\mathrm{H}}^{(1)}+C P_{\mathrm{H}}^{(1)}+D_{1}(1-\lambda)+E\right]^{2}}
$$

where

$$
\begin{aligned}
f_{1}(\lambda)= & \frac{a E_{\max }\left(e^{a b}+1\right) \exp (-a \lambda K)}{[1+\exp (a b-a \lambda K)]^{2}} \\
f_{2}(\lambda)= & \frac{C E_{\max }\left(1-e^{-a \lambda K}\right)^{2} e^{a \lambda K}}{a\left(e^{a b}+1\right)}-D_{1}(1-\lambda)^{2} \\
& +E \frac{\left(e^{a b}+e^{a \lambda K}\right)\left(1-e^{-a \lambda K}\right)}{a\left(e^{a b}+1\right)}-E(1-\lambda) .
\end{aligned}
$$

Let $\partial \gamma_{r d}^{(1)} / \partial \lambda=0$ and we have $f_{1}(\lambda) f_{2}(\lambda)=0$. Since $f_{1}(\lambda)>0$ always holds, $\partial \gamma_{r d}^{(1)} / \partial \lambda=0$ is equivalent to $f_{2}(\lambda)=$ 0 . Further, taking the first-order derivative of $f_{2}(\lambda)$, we have

$$
\begin{aligned}
\frac{\partial f_{2}(\lambda)}{\partial \lambda}= & \frac{C E_{\max } K e^{a \lambda K}\left(1-e^{-2 a \lambda K}\right)}{e^{a b}+1}+2 D_{1}(1-\lambda) \\
& +E+\frac{E K\left(e^{a b-a \lambda K}+e^{a \lambda K}\right)}{e^{a b}+1}>0 .
\end{aligned}
$$

Thus, $f_{2}(\lambda)$ increases with the increasing of $\lambda$. Since both $f_{2}(0)=-D_{1}-E<0$ and $f_{2}(1)=C E_{\max }\left(1-e^{-a K}\right)^{2} e^{a K} / a\left(e^{a b}+\right.$ $1)+E\left(e^{a b}+e^{a K}\right)\left(1-e^{-a K}\right) / a\left(e^{a b}+1\right)>0$ are satisfied, there exists a unique $\lambda \in[0,1]$ for $f_{2}(\lambda)=0$.

Let $s_{1}$ denote the solution to $f_{2}(\lambda)=0$. Then $s_{1}$ is also the solution to $\partial \gamma_{r d}^{(1)} / \partial \lambda=0$. Based on the expression of $\partial \gamma_{r d}^{(1)} / \partial \lambda, \gamma_{r d}^{(1)}$ increases with increasing $\lambda$ when $\lambda \epsilon$ $\left[0, s_{1}\right]$ and decreases with increasing $\lambda$ for $\lambda \in\left[s_{1}, 1\right]$. Therefore, $\gamma_{r d}^{(1)}$ is a unimodal function and $s_{1}$ is the optimal solution to $\mathscr{P} 2$. Here, the value of $s_{1}$ can be determined by means of the golden section search method. The details of this method are summarized in Algorithm 1. According to [21], the computational complexity of this algorithm can be computed as $O(\log (1 / \varepsilon))$, where $\varepsilon$ is the precision.

3.2. Nonlinear EH Model Based on the Inverse Proportional Function. In this subsection, we design an optimal PS scheme to maximize the overall system capacity for an AF relay system with SWIPT based on the nonlinear EH model in [16]. Based on (2), the received SNR from $R$ to $D$ under the nonlinear EH model, in (2), is given by

$$
\gamma_{r d}^{(2)}=\frac{A(1-\lambda) P_{\mathrm{H}}^{(2)}}{B\left[(1-\lambda) P_{\mathrm{H}}^{(2)}+C P_{\mathrm{H}}^{(2)}+D_{1}(1-\lambda)+E\right]} .
$$

Then the optimization problem can be formulated as

$$
\begin{aligned}
\mathscr{P} 3: \text { maximize } & C_{\text {total }}^{(2)} \\
\text { subject to } & 0 \leq \lambda \leq 1,
\end{aligned}
$$

where $C_{\text {total }}^{(2)}=(T / 2) \log _{2}\left(1+\gamma_{s d}+\gamma_{r d}^{(2)}\right)$. Similar to $\mathscr{P} 1, \mathscr{P} 3$ can be reformulated as

$$
\begin{aligned}
\mathscr{P} 4: \text { maximize } & \gamma_{r d}^{(2)} \\
\text { subject to } & 0 \leq \lambda \leq 1 .
\end{aligned}
$$




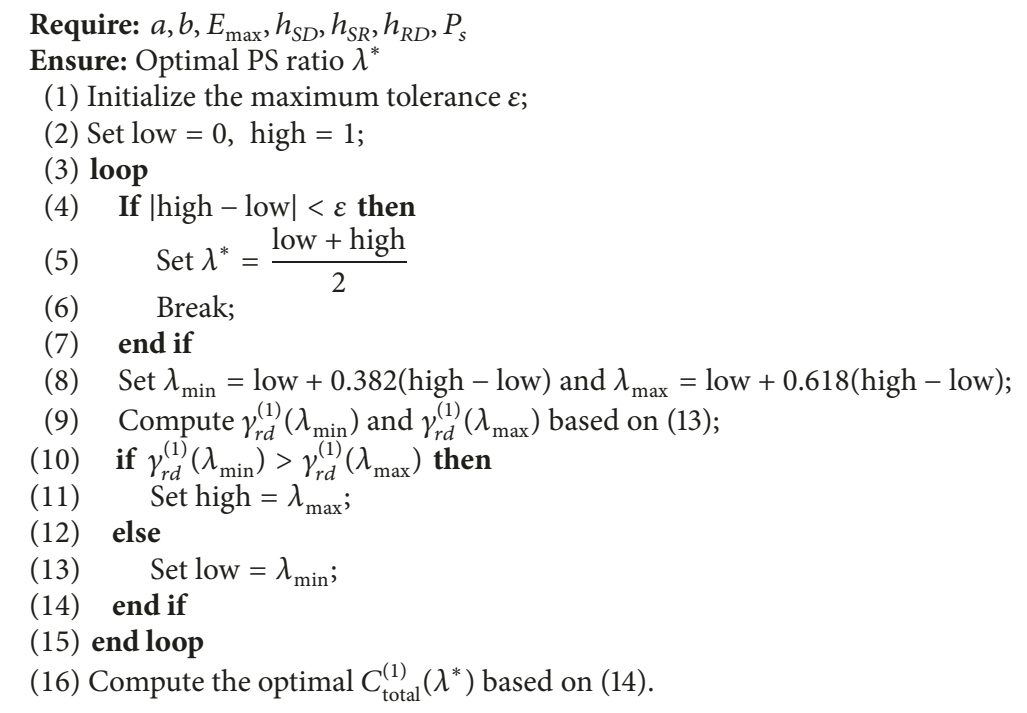

Algorithm 1: Golden section search algorithm.

According to (20), the first-order derivative of $\gamma_{r d}^{(2)}$ with respect to $\lambda$ is

$$
\frac{\partial \gamma_{r d}^{(2)}}{\partial \lambda}=\frac{A f_{3}(\lambda)\left(M \lambda^{2}-N \lambda+Q\right)}{B\left[(1-\lambda) P_{\mathrm{H}}^{(2)}+C P_{\mathrm{H}}^{(2)}+D_{1}(1-\lambda)+E\right]^{2}}
$$

where $f_{3}(\lambda)=c(a-b / c) /(x+c)^{2}, M=D_{1}-C K(a c-b) / c^{2}-$ $E K / c, N=2 D_{1}+2 E$, and $Q=D_{1}+E$.

Based on (2), we have

$$
\lim _{\lambda K \longrightarrow+\infty}\left(\frac{a \lambda K+b}{\lambda K+c}-\frac{b}{c}\right)=a-\frac{b}{c}>0 .
$$

Therefore, $f_{3}(\lambda)>0$ always holds for any value of $\lambda \in[0$, 1].

Let $\partial \gamma_{r d}^{(2)} / \partial \lambda=0$ and we have $M \lambda^{2}-N \lambda+Q=0$. Thus, we need to obtain the solutions to $M \lambda^{2}-N \lambda+Q=0$ to achieve the optimal PS ratio.

According to the expression of $M$, there are two cases for the solutions to $M \lambda^{2}-N \lambda+Q=0$, which are Case 1: $M=0$; Case 2: $M \neq 0$.

Case 1. Based on $M=0$, we have $-N \lambda+Q=0$. Then the solution to $M \lambda^{2}-N \lambda+Q=0$ is given by $Q / N$ and $Q / N=$ 0.5 . Since $\gamma_{r d}^{(2)}$ increases with increasing $\lambda$ when $\lambda \in[0,0.5]$ and decreases with increasing $\lambda$ for $\lambda \in[0.5,1]$, the optimal solution to $\mathscr{P} 4$ is 0.5 and $\lambda^{*}=0.5$.

Case 2. Let $\Delta=N^{2}-4 M Q$. When both $\Delta<0$ and $M>0$ are satisfied, $M \lambda^{2}-N \lambda+Q>0$ always holds and $\partial \gamma_{r d}^{(2)} / \partial \lambda>0$ holds for any value of $\lambda \in[0,1]$. Therefore, $\gamma_{r d}^{(2)}$ increases with the increasing of $\lambda$ and the optimal PS ratio $\lambda^{*}$ is 1 .
When $\Delta \geq 0$ is satisfied, the solutions to $M \lambda^{2}-N \lambda+Q=0$ are given by

$$
\begin{aligned}
& x_{1}=\frac{N-\sqrt{\Delta}}{2 M}, \\
& x_{2}=\frac{N+\sqrt{\Delta}}{2 M} .
\end{aligned}
$$

Let $x_{\min }=\min \left(x_{1}, x_{2}\right)$ and $x_{\max }=\max \left(x_{1}, x_{2}\right)$.

For the case with $M>0$, we have $x_{\min }>0$ and $x_{\max }>0$. (i) If $x_{\max }<1$ is satisfied, $\gamma_{r d}^{(2)}$ increases with $\lambda \epsilon$ $\left[0, x_{\min }\right]$, decreases with $\lambda \in\left[x_{\min }, x_{\max }\right]$, and then increases with $\lambda \in\left[x_{\max }, 1\right]$. The maximum $\gamma_{r d}^{(2)}$ can be obtained by $\max \left(\gamma_{r d}^{(2)}\left(x_{\min }\right), \gamma_{r d}^{(2)}(1)\right)$. Since $\gamma_{r d}^{(2)}\left(x_{\min }\right)>\gamma_{r d}^{(2)}(0)=0=$ $\gamma_{r d}^{(2)}(1)$ holds, the optimal PS ratio is given by $\lambda^{*}=x_{\min }$. (ii) If $x_{\min }>1$ is satisfied, $\gamma_{r d}^{(2)}$ is a monotonic increasing function and the optimal solution to $\mathscr{P} 4$ is $\lambda^{*}=1$. (iii) If $x_{\min } \leq 1 \leq x_{\text {max }}$ is satisfied, $\gamma_{r d}^{(2)}$ increases with $\lambda \in\left[0, x_{\min }\right]$ and decreases with $\lambda \in\left[x_{\min }, 1\right]$. In this case, the optimal PS ratio is given by $\lambda^{*}=x_{\min }$.

For the case with $M<0$, we have $x_{\min }<0$ and $x_{\max }>0$. (i) If $x_{\max }>1$ is satisfied, $\partial \gamma_{r d}^{(2)} / \partial \lambda>0$ always holds for $\lambda \epsilon$ $[0,1] \cdot \gamma_{r d}^{(2)}$ increases with the increasing of $\lambda$ and the optimal PS ratio is given by $\lambda^{*}=1$. (ii) If $x_{\max } \leq 1$ is satisfied, $\gamma_{r d}^{(2)}$ increases with $\lambda \in\left[0, x_{\max }\right]$ and decreases with $\lambda \in\left[x_{\max }, 1\right]$. In this case, the optimal PS ratio is given by $\lambda^{*}=x_{\max }$.

Thus, the optimal solution to $\mathscr{P} 3$ is summarized as Proposition 1.

Proposition 1. The optimal PS ratio for $\mathscr{P} 3$ is given by

$$
\lambda^{*}= \begin{cases}0.5, & \text { for } M=0 \\ x_{\min }, & \text { for } M>0, \Delta \geq 0 \text { and } x_{\min }<1 \\ x_{\max }, & \text { for } M<0, \Delta \geq 0 \text { and } x_{\max }<1 \\ 1, & \text { otherwise. }\end{cases}
$$




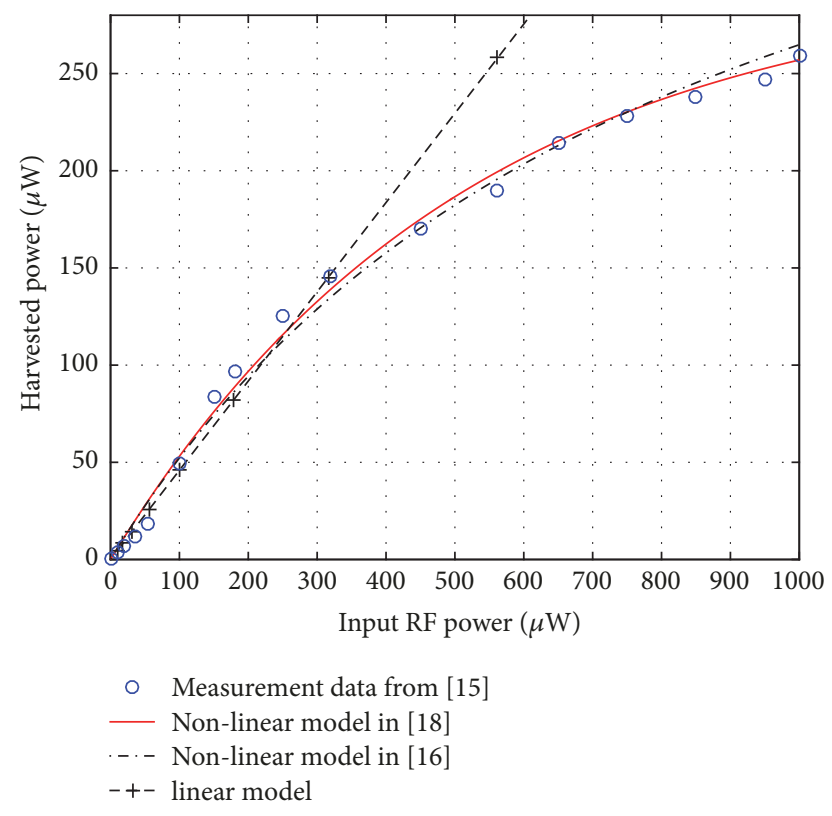

FIGURE 2: Comparison between the harvested powers among the nonlinear $\mathrm{EH}$ models in $[16,18]$, the linear $\mathrm{EH}$ model, and the experimental data from [15].

\section{Numerical Results and Discussions}

In this section, we evaluate the performance of the designed PS schemes based on two nonlinear EH models: the nonlinear $\mathrm{EH}$ model based on the logistic function and the nonlinear $\mathrm{EH}$ model based on the inverse proportional function, respectively. The basic parameters throughout the simulations, unless otherwise specified, are as follows. We consider the path model to be $\left|h_{i j}\right|^{2}=\left|g_{i j}\right|^{2} d_{i j}^{-\alpha_{i j}}$ (ij=SR, SD or RD), where $d_{i j}$ is the $i-j$ distance, $\left|g_{i j}\right|^{2}$ denotes the $i-j$ small-scale fast fading, and $\alpha_{i j}$ is the $i-j$ path loss exponent. Further, we assume that $g_{S D}$ and $g_{R D}$ follow the quasi-static Rayleigh fading with unit variance, and that $g_{S R}$ follows the Rician fading with the Rician factor $3+\sqrt{12}$. We set $d_{S D}=20$ meters, $d_{S R}=2$ meters, $d_{R D}=d_{S D}-d_{S R}, \alpha_{S R}=\alpha_{R D}=3$, and $\alpha_{S D}=4$. The channel noise power is set to be $\sigma_{s r}^{2}=0.0001$, $\sigma_{p}^{2}=0.001$, and $\sigma_{r d}^{2}=\sigma_{s d}^{2}=-40 \mathrm{dBm}$, respectively.

Figure 2 compares the experimental data in [15] with the two nonlinear EH models and the conventional linear $\mathrm{EH}$ model. The parameters of the nonlinear model based on the logistic function are $a=1.953 \times 10^{-3}, b=-3.571 \times 10^{3}$, and $E_{\max }=299.5 \mu \mathrm{W}$. The parameters for the nonlinear $\mathrm{EH}$ model based on the inverse proportional function are $a=2.463, b=1635$, and $c=826$. For the linear EH model, the conversion efficiency is set to be 0.4591 . As shown in this figure, compared with the linear $\mathrm{EH}$ model, the nonlinear models in $[16,18]$ are more accurate when modelling the practical RF-DC circuits. The root mean squared errors of the nonlinear model based on the logistic function, the nonlinear model based on the inverse proportional function, and the linear model are 9.5554 $\mu \mathrm{W}, 11.2464 \mu \mathrm{W}$, and $73.6854 \mu \mathrm{W}$,

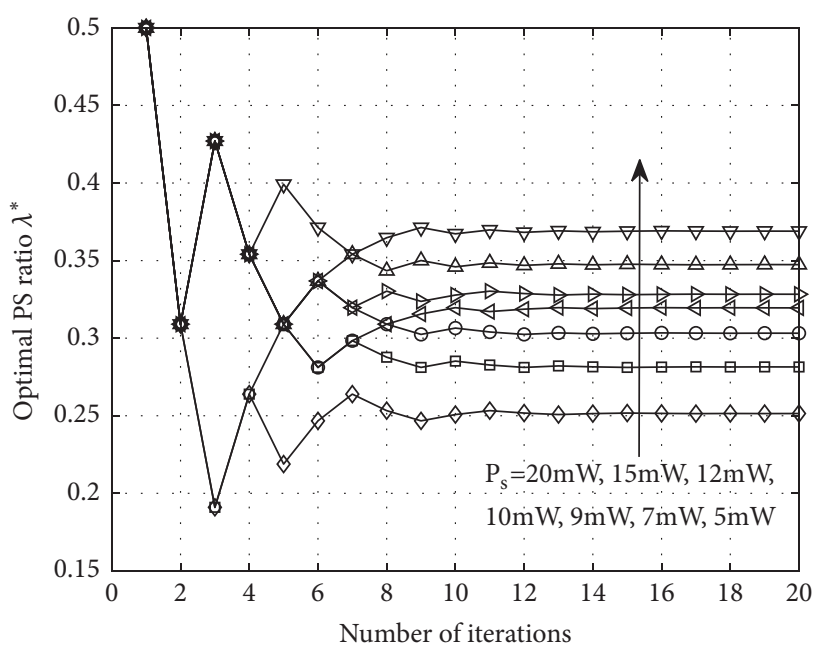

Figure 3: Convergence of Algorithm 1 under different sets of $P_{s}$.

respectively. The results show that the nonlinear model in [18] is the most accurate among them, and that the nonlinear model in [16] is also able to provide sufficient precision.

Figure 3 demonstrates the convergence of the Algorithm 1 for different sets of the transmit powers. The maximum tolerance $\varepsilon$ is set to be 0.0001 . From this figure, we can see that the optimal PS ratio $\lambda^{*}$ always converges to the optimal value within a limited number of iterations. For example, for the case with $P_{s}=10 \mathrm{~mW}$, the optimal PS ratio is obtained when the number of iterations is 11. Another observation is that the optimal PS ratio decreases with the increasing of $P_{s}$. The reason is as follows. With a given PS ratio, the input power of the energy harvester increases with the increasing of the transmit power. When the transmit power is large enough, the energy harvester is always saturated and more energy will be wasted. As a result, the optimal PS ratio decreases with the increasing $P_{s}$ to avoid the waste of energy.

The PS schemes used for comparing are as follows: (1) the proposed PS scheme with the nonlinear EH model based on the logistic function where the optimal PS ratio is obtained by Algorithm 1; (2) the proposed PS scheme with the nonlinear $\mathrm{EH}$ model based on the inverse proportional function where the optimal PS ratio is determined by Proposition 1; (3) baseline scheme where the conventional linear EH model is employed. For convenience, we denote the proposed PS scheme with the nonlinear EH model based on the logistic function as LFPS and the proposed PS scheme with the nonlinear $\mathrm{EH}$ model based on the inverse proportional function as IPFPS. Since the nonlinear EH model based on the logistic function is closest to the practical EH circuit in [15], we use it to model the practical EH circuit. The optimal PS ratios obtained by LFPS, IPFPS, and baseline scheme will be applied to the practical EH circuit to investigate the performance of LFPS, IPFPS, and baseline schemes under the practical EH circuit. On this basis, the corresponding system capacities can be determined.

Figure 4 presents the overall system capacity achieved by the LFPS, IPFPS, and baseline schemes varying with the 




FIGURE 4: System capacity versus the transmit power $P_{s}$.

transmit power. It can be observed that, for all considered schemes, the system capacity increases monotonically as we increase the transmit power $P_{s}$ at the source. Both LFPS and IPFPS schemes outperform the baseline scheme and LFPS can achieve a higher system capacity than IPFPS. This is because of the fact that the mismatches between the $\mathrm{EH}$ models and the practical $\mathrm{EH}$ circuits will lead to resource allocation mismatches which will bring a poor performance. The more accurate the energy harvesting model, the better the performance the PS scheme can achieve. These results also match the results in Figure 2 perfectly. Besides, we can also see that the performance gain between LFPS and IPFPS or baseline scheme increases with the increasing of $P_{s}$. This is due to the fact that, with the increasing of $P_{s}$, the gap between the nonlinear EH model based on the inverse proportional function or the linear $\mathrm{EH}$ model and the practical energy harvester becomes larger, leading to a worse perform ance.

\section{Conclusion}

In this paper, we have developed the optimal PS schemes for an AF relay system with direct link to maximize the system capacity, where both the nonlinear EH model based on the logistic function and the nonlinear $\mathrm{EH}$ model based on the inverse proportional function are employed to model the practical energy harvester. Through simulations, three insights were obtained as follows: (1) the PS schemes based on the nonlinear models can achieve superior performance than the one based on the linear model; (2) the PS scheme optimized for the former nonlinear $\mathrm{EH}$ model can achieve the best performance, while an extra computational complexity to obtain the optimal solution is incurred; (3) for the case with the latter nonlinear EH model, the PS scheme can achieve near-optimal performance with low computational complexity.

\section{Data Availability}

The data used to support the findings of this study are available from the corresponding author upon request.

\section{Conflicts of Interest}

The authors declare that they have no conflicts of interest.

\section{Acknowledgments}

This work was supported by the Research Program of Education Bureau of Shaanxi Province (17JK0699), Shaanxi Industrial Science and Technology Key Project (2017GY039), and 2017 China Textile Industry Federation Science and Technology Guidance Project (2017067).

\section{References}

[1] Z. Chu, F. Zhou, Z. Zhu, R. Q. Hu, and P. Xiao, "Wireless Powered Sensor Networks for Internet of Things: Maximum Throughput and Optimal Power Allocation," IEEE Internet of Things Journal, vol. 5, no. 1, pp. 310-321, Feb, 2018.

[2] L. Fan, X. Lei, N. Yang, T. Q. Duong, and G. K. Karagiannidis, "Secure Multiple Amplify-and-Forward Relaying with Cochannel Interference," IEEE Journal of Selected Topics in Signal Processing, vol. 10, no. 8, pp. 1494-1505, 2016.

[3] L. Fan, X. Lei, N. Yang et al., "Secrecy cooperative networks with outdated relay selection over correlated fading channels," IEEE Transactions on Vehicular Technology, vol. 66, no. 8, pp. 75997603, 2017.

[4] A. A. Nasir, X. Zhou, S. Durrani, and R. A. Kennedy, "Relaying protocols for wireless energy harvesting and information processing," IEEE Transactions on Wireless Communications, vol.12, no. 7, pp. 3622-3636, 2013.

[5] Y. Ye, Y. Li, D. Wang, F. Zhou, R. Q. Hu, and H. Zhang, "Optimal Transmission Schemes for DF Relaying Networks Using SWIPT,' IEEE Transactions on Vehicular Technology, pp. $1-8,2018$.

[6] Y. Ye, Y. Li, Z. Wang, X. Chu, and H. Zhang, "Dynamic Asymmetric Power Splitting Scheme for SWIPT-Based TwoWay Multiplicative AF Relaying," IEEE Signal Processing Letters, vol. 25, no. 7, pp. 1014-1018, 2018.

[7] A. A. Nasir, X. Zhou, S. Durrani, and R. A. Kennedy, “Throughput and ergodic capacity of wireless energy harvesting based DF relaying network," in Proceedings of the IEEE International Conference on Communications (ICC '14), pp. 4066-4071, Sydney, Australia, June 2014.

[8] M. Ju, K.-M. Kang, K.-S. Hwang, and C. Jeong, "Maximum transmission rate of PSR/TSR protocols in wireless energy harvesting DF-based relay networks," IEEE Journal on Selected Areas in Communications, vol. 33, no. 12, pp. 2701-2717, 2015.

[9] S. Atapattu and J. Evans, "Optimal energy harvesting protocols for wireless relay networks," IEEE Transactions on Wireless Communications, vol. 15, no. 8, pp. 5789-5803, 2016.

[10] L. Hu, C. Zhang, and Z. Ding, "Dynamic power splitting policies for AF relay networks with wireless energy harvesting," in Proceedings of the 2015 ICC - 2015 IEEE International Conference on Communications Workshops (ICC), pp. 2035-2039, London, UK, June 2015.

[11] H. Lee, C. Song, S. Choi, and I. Lee, "Outage probability analysis and power splitter designs for swipt relaying systems with direct 
link," IEEE Communications Letters, vol. 21, no. 3, pp. 648-651, 2017.

[12] I. Krikidis, "Simultaneous information and energy transfer in large-scale networks with/without relaying," IEEE Transactions on Communications, vol. 62, no. 3, pp. 900-912, 2014.

[13] Y. Liu, Z. Ding, M. Elkashlan, and H. V. Poor, "Cooperative Non-orthogonal Multiple Access with Simultaneous Wireless Information and Power Transfer," IEEE Journal on Selected Areas in Communications, vol. 34, no. 4, pp. 938-953, 2016.

[14] Y. Ye, Y. Li, D. Wang, and G. Lu, "Power splitting protocol design for the cooperative NOMA with SWIPT," in Proceedings of the IEEE International Conference on Communications, pp. 1-5, May 2017.

[15] T. Le, K. Mayaram, and T. Fiez, "Efficient far-field radio frequency energy harvesting for passively powered sensor networks," IEEE Journal of Solid-State Circuits, vol. 43, no. 5, pp. 1287-1302, 2008.

[16] Y. Chen, N. Zhao, and M. Alouini, "Wireless Energy Harvesting Using Signals From Multiple Fading Channels," IEEE Transactions on Communications, vol. 65, no. 11, pp. 5027-5039, 2017.

[17] C. R. Valenta and G. D. Durgin, "Harvesting wireless power: survey of energy-harvester conversion efficiency in far-field, wireless power transfer systems," IEEE Microwave Magazine, vol. 15, no. 4, pp. 108-120, 2014.

[18] E. Boshkovska, D. W. K. Ng, N. Zlatanov, and R. Schober, "Practical non-linear energy harvesting model and resource allocation for SWIPT systems," IEEE Communications Letters, vol. 19, no. 12, pp. 2082-2085, 2015.

[19] D. Mishra, S. De, and D. Krishnaswamy, "Dilemma at RF Energy Harvesting Relay: Downlink Energy Relaying or Uplink Information Transfer?" IEEE Transactions on Wireless Communications, vol. 16, no. 8, pp. 4939-4955, Aug, 2017.

[20] F. Zhou, Z. Chu, H. Sun, R. Q. Hu, and L. Hanzo, "Artificial Noise Aided Secure Cognitive Beamforming for Cooperative MISO-NOMA Using SWIPT," IEEE Journal on Selected Areas in Communications, pp. 1-1, 2018.

[21] L. Shi, L. Zhao, K. Liang, X. Chu, G. Wu, and H. Chen, "Profit maximization in wireless powered communications with improved non-linear energy conversion and storage efficiencies," in Proceedings of the IEEE International Conference on Communications, pp. 1-6, May, 2017.

[22] H. Niu, D. Guo, Y. Huang, and B. Zhang, "Robust Energy Efficiency Optimization for Secure MIMO SWIPT Systems With Non-Linear EH Model," IEEE Communications Letters, vol. 21, no. 12, pp. 2610-2613, 2017.

[23] Q. Yao, T. Q. S. Quek, A. Huang, and H. Shan, "Joint Downlink and Uplink Energy Minimization in WET-Enabled Networks," IEEE Transactions on Wireless Communications, vol. 16, no. 10, pp. 6751-6765, Oct, 2017.

[24] Y. Wang, Y. Wang, F. Zhou, Y. Wu, and H. Zhou, "Resource allocation in wireless powered cognitive radio networks based on a practical non-linear energy harvesting model," IEEE Access, vol. 5, pp. 17618-17626, 2017.

[25] J.-M. Kang, I.-M. Kim, and D. I. Kim, "Mode Switching for SWIPT over Fading Channel with Nonlinear Energy Harvesting," IEEE Wireless Communications Letters, vol. 6, no. 5, pp. 678-681, Oct, 2017.

[26] S. Wang, M. Xia, K. Huang, and Y. Wu, "Wirelessly Powered Two-Way Communication With Nonlinear Energy Harvesting Model: Rate Regions Under Fixed and Mobile Relay,' IEEE Transactions on Wireless Communications, vol. 16, no. 12, pp. 8190-8204, 2017.
[27] T. Wang, G. Lu, Y. Ye, and Y. Ren, "Dynamic Power Splitting Strategy for SWIPT Based Two-Way Multiplicative AF Relay Networks with Nonlinear Energy Harvesting Model," Wireless Communications and Mobile Computing, vol. 2018, Article ID 1802063, 9 pages, 2018.

[28] A. Cvetkovic and V. Blagojevic, "Performance Analysis of Nonlinear Energy-Harvesting DF Relay System in InterferenceLimited Nakagami-m Fading Environment," ETRI Journal, vol. 39, no. 6, pp. 803-812, Dec, 2017.

[29] J. Zhang and G. Pan, "Outage Analysis of Wireless-Powered Relaying MIMO Systems with Non-Linear Energy Harvesters and Imperfect CSI," IEEE Access, vol. 4, pp. 7046-7053, 2016.

[30] K. Wang, Y. Li, Y. Ye, and H. Zhang, "Dynamic Power Splitting Schemes for Non-Linear EH Relaying Networks: Perfect and Imperfect CSI," in Proceedings of the IEEE 86th Vehicular Technology Conference, pp. 1-5, Sept, 2017.

[31] L. Wei, R. Q. Hu, Y. Qian, and G. Wu, "Energy efficiency and spectrum efficiency of multihop device-to-device communications underlaying cellular networks," IEEE Transactions on Vehicular Technology, vol. 65, no. 1, pp. 367-380, 2016. 




\section{Enfincering}
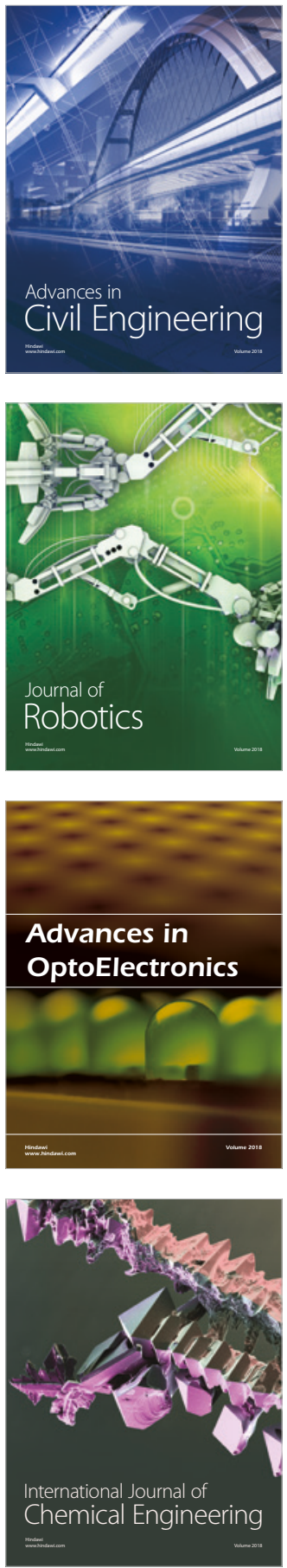

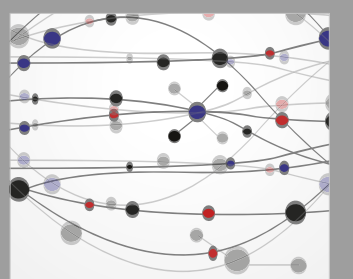

\section{Rotating \\ Machinery}

The Scientific World Journal

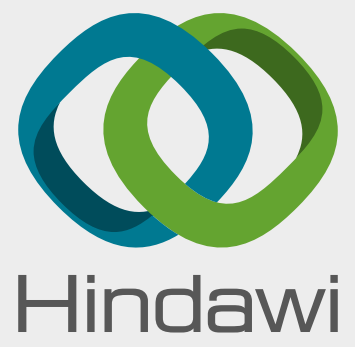

Submit your manuscripts at

www.hindawi.com

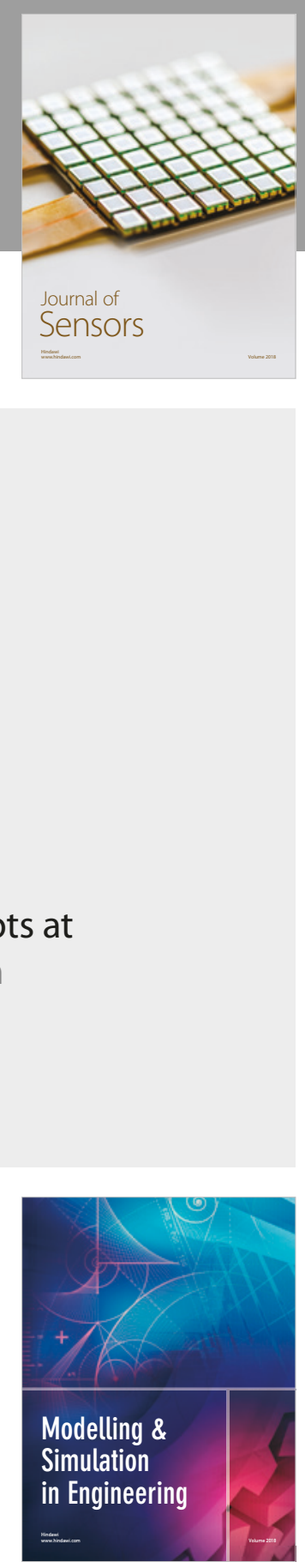

\section{Advances \\ Multimedia}
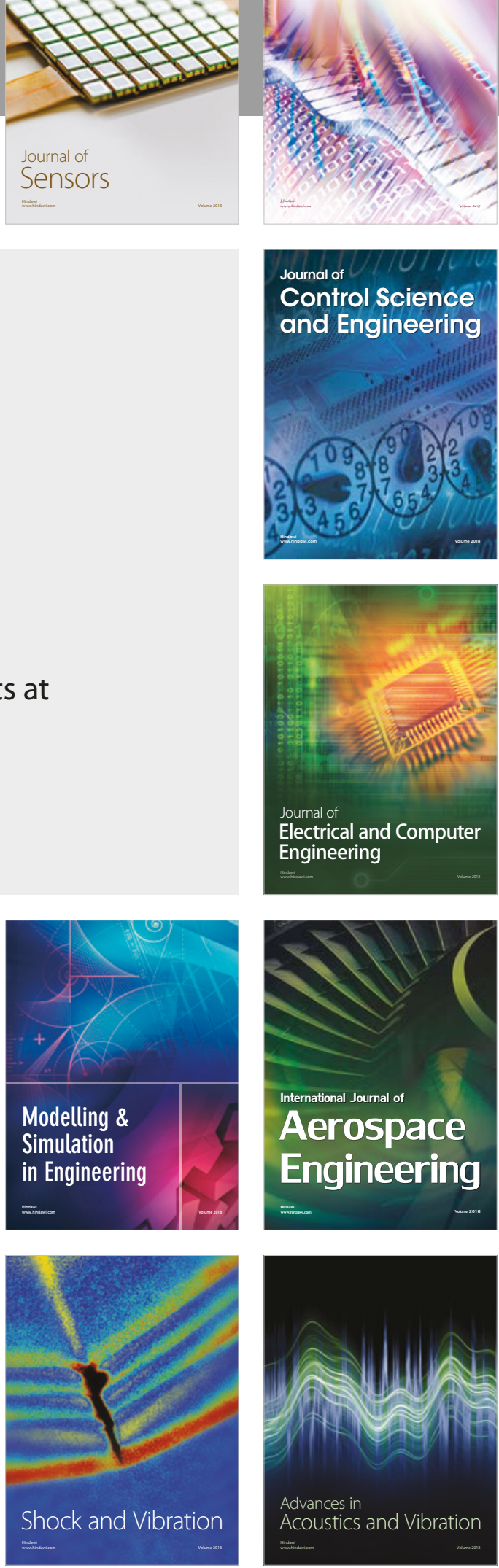